\title{
A FUNCTOR TO RINGED SPACES ${ }^{1}$
}

\author{
GAIL L. CARNS
}

\begin{abstract}
With the set of orders $\mathcal{O}$ on a field and the Harrison topology induced from the set of all primes as a base space we define a ringed space $(\mathcal{O}, \mathcal{F})$. For each field homomorphism we find an associated ringed space morphism producing a contravariant functor from the category of fields to the category of ringed spaces. An equivalence relation $\sim$ is defined on the set of orders and again a ringed space $(\mathcal{O} / \sim, \tilde{F})$ and a contravariant functor from fields to ringed spaces is obtained along with a natural transformation from the first to the second functor. Finally, we obtain a ringed space morphism $(\mathcal{O} / \sim, \overline{\mathcal{F}}) \rightarrow\left(Y, \mathcal{\Theta}_{Y}\right)$ where $Y$ is the spectrum of the ring of bounded elements and $\Theta_{Y}$ is the structure sheaf.
\end{abstract}

1. Introduction. This paper is concerned with the set of orders $\mathcal{O}$ on a field $F$ with the topology induced from the Harrison topology on the set of all primes. With $\theta$ as the base space we shall define a sheaf of rings $\mathfrak{F}$. Thus for each field we have an associated ringed space $(\mathcal{O}, \mathfrak{F})$. For each field homomorphism we find an associated ringed space morphism producing a contravariant functor from the category of fields to the category of ringed spaces. The reason for using the Harrison topology is that this is the topology which guarantees the functions used in defining the sheaf will be continuous. Next, an equivalence relation $\sim$ is defined on $\theta$ and we obtain another ringed space $(\Theta / \sim, \bar{F})$ and another contravariant functor. We also find a ringed space morphism $(\mathcal{O}, \mathcal{F}) \rightarrow(\mathcal{O} / \sim, \bar{F})$ producing a natural transformation from the first functor to the second. Finally, we obtain a ringed space morphism $(\Theta / \sim, \bar{F}) \rightarrow\left(Y, \mathcal{O}_{Y}\right)$ where $Y$ is the spectrum of the ring of bounded elements of $F$ and $\mathcal{O}_{Y}$ is the structure sheaf. We will use the definitions and notations of [8, Chapters 3 and 4$]$.

2. A note on ringed space morphisms. The ringed spaces to be considered in this section are those where the sheaves are sheaves of functions to rings, the restriction maps are the restriction maps in the usual sense, and the rings of functions are equipped with pointwise operations. All definitions in this section will be taken from [5].

Received by the editors October 21, 1970.

AMS 1969 subject classifications. Primary 0690, 1270.

Key words and phrases. Field, sheaf, ringed space, order, structure sheaf.

1 This paper is part of a dissertation written under the direction of D. W. Dubois at the University of New Mexico. 
Proposition A. Let $X$ and $Y$ be topological spaces, $\psi: X \rightarrow Y$ a continuous map, and $\left(X, \mathcal{F}_{X}\right)$ and $\left(Y, \mathcal{F}_{Y}\right)$ ringed spaces. Suppose that $\psi$ has the property that if $f \in \mathcal{F}_{Y}(U)$ then $\left.f \psi\right|_{\psi^{-1}(U)} \in \psi^{-1} \mathfrak{F}_{X}(U)$ where $\psi^{-1} \mathcal{F}_{X}$ denotes the direct image of $\mathcal{F}_{X}$ under $\psi$. Define $\theta(U): \mathcal{F}_{Y}(U)$ $\rightarrow \psi^{-1} \mathcal{F}_{X}(U)$ by $\theta(U)(f)=f \psi$ and let $\theta=\{\theta(U): U$ in open $Y\}$. Then $(\psi, \theta):\left(Y, \mathcal{F}_{Y}\right) \rightarrow\left(X, \mathcal{F}_{X}\right)$ is a ringed space morphism.

The proof is straightforward.

3. The sheaf $\mathcal{F}$. Let $F$ be a field and $\mathcal{O}$ the set of orders on $F$ where an order is a subset $\alpha$ that is closed under multiplication, addition, and division such that $\alpha \cap-\alpha=\{0\}$ and $\alpha \cup-\alpha=F$. The Harrison topology on $\theta$ is the topology for which

$$
\left\{X_{G}=\{\alpha \in \Theta: G \cap \alpha=\varnothing\}: G \text { is a finite subset of } F\right\}
$$

is a base. lt is well known [6] that the Harrison topology on the set of all primes is compact. Also the set of orders forms a closed subset of the set of primes hence $\mathcal{O}$ is a compact topological space.

Recall that if a field $F$ is orderable then the field $Q$ of rational numbers is a subfield of $F$. Thus for $\alpha \in \mathcal{O}$ and $b \in F$ we can define $(b, \alpha)$ $=\sup \{r \in Q: b-r \in \alpha\}$. Note that for fixed $\alpha$ this defines a place $F \rightarrow R$ with valuation ring $B_{\alpha}$. Let $U$ be an open subset of $\mathcal{O}$, a real valued function $f: U \rightarrow R$ is said to be good on $U$ if there exists $b \in F$ such that $f(\alpha)=(b, \alpha)$ for all $\alpha \in U$. A function $f: U \rightarrow R$ is said to be locally good on $U$ if for each $\alpha \in U$ there exists $V_{\alpha}$ open in $U$ such that $f$ restricted to $V_{\alpha}$ is good on $V_{\alpha}$ and $\alpha \in V_{\alpha}$.

Proposition. If $f: U \rightarrow R$ is locally good then $f$ is continuous.

Proof. Clearly it suffices to prove that $f$ good on $U$ implies $f$ is continuous thus we assume that $f(\alpha)=(b, \alpha)$ for all $\alpha \in U$. Since $(b, \alpha)$ is linear in the first coordinate [2] all we need to show is that $f^{-1}(0, \infty)$ is open in $U$.

$$
\begin{aligned}
f^{-1}(0, \infty) & =\{\alpha \in U: b-1 / n \in \alpha \text { for some } n \geqq 0\} \\
& =\{\alpha \in \Theta: b-1 / n \in \alpha \text { for some } n \geqq 0\} \cap U \\
& =\bigcup_{n=1}^{\infty}\{\alpha \in 0: b-1 / n \in \alpha\} \cap U \\
& =\bigcup_{n=1}^{\infty}\{\alpha \in 0: 1 / n-b \notin \alpha\} \cap U \text { which is open. }
\end{aligned}
$$

We shall now define a sheaf of rings, $\mathcal{F}$, on $\mathcal{O}$ by letting $\mathcal{F}(U)$ $=\{f: f$ is locally good on $U\}$ and for $U \subseteq V$ the restriction map 
$\mathscr{F}(V) \rightarrow \mathscr{F}(U)$ will be defined to be the restriction map in the usual sense.

TheOREM 1. F, defined above, is a sheaf of rings where $\mathcal{F}(U)$ is equipped with pointwise operations.

Proof. To prove that $\mathcal{F}(U)$ is a ring for each $U$ open in $\mathcal{O}$ we need only prove that if $f_{1}, f_{2} \in \mathcal{F}(U)$ then $f_{1}+f_{2}, f_{1} f_{2} \in \mathcal{F}(U)$. The other conditions are routine verifications. For $1 \leqq i \leqq 2$ let $f_{i} \in \mathcal{F}(U)$ and $\beta \in U$ then there exists $V_{i}$ open in $U$ and $b_{i} \in F$ such that $\beta \in V_{i}$ and $f_{i}(\alpha)=\left(b_{i}, \alpha\right)$ for all $\alpha \in V_{i}$. Then $\beta \in V=V_{1} \cap V_{2}$ is open in $U$ and $\left(f_{1}+f_{2}\right)(\alpha)=f_{1}(\alpha)+f_{2}(\alpha)=\left(b_{1}, \alpha\right)+\left(b_{2}, \alpha\right)=\left(b_{1}+b_{2}, \alpha\right)$ for all $\alpha \in V$, also $\left(f_{1} f_{2}\right)(\alpha)=f_{1}(\alpha) f_{2}(\alpha)=\left(b_{1}, \alpha\right)\left(b_{2}, \alpha\right)=\left(b_{1} b_{2}, \alpha\right)$ for all $\alpha \in V$ hence $f_{1}+f_{2}, f_{1} f_{2} \in \mathcal{F}(U)$.

To prove that $\mathcal{F}$ is a sheaf let $\left(U_{i}\right)$ be an open cover of $U$ and $\left(f_{i}\right)$ a family such that $f_{i} \in \mathcal{F}\left(U_{i}\right)$ and $f_{i}$ and $f_{j}$ have the same restriction to $U_{i} \cap U_{j}$ for all $i$ and $j$. We need to find a unique $f \in \mathcal{F}(U)$ such that $f$ restricted to $U_{i}$ is $f_{i}$. The only possible definition for $f$ is $f(\alpha)=f_{i}(\alpha)$ where $\alpha \in U_{i}$ hence we need to show that the $f$ just defined is locally good on $U$. Let $\alpha \in U$, then there exists $i$ such that $\alpha \in U_{i}$ and hence there exists $V$ open in $U_{i}$ (and thus open in $U$ ) and $b \in F$ such that $\alpha \in V$ and $f(\beta)=f_{i}(\beta)=(b, \beta)$ for all $\beta \in V$. Thus $f$ is locally good on $U$ completing the proof.

It should be pointed out that in no part of the proof of Theorem 1 did we need to know which topology we were considering. Hence for any topology on $\mathcal{O}$ the presheaf of locally good functions is a sheaf of rings.

If we now let $h: F_{1} \rightarrow F_{2}$ be a field homomorphism we obtain an induced $\psi: \theta_{2} \rightarrow \Theta_{1}$ defined by $\psi(\alpha)=h^{-1}(\alpha)$ where $\theta_{1}$ and $\theta_{2}$ are the set of orders on $F_{1}$ and $F_{2}$ respectively.

LEMмA. $\psi$, defined above, is a continuous map.

Let $\digamma_{1}$ and $\digamma_{2}$ be the sheaves of locally good functions on $\mathcal{O}_{1}$ and $\theta_{2}$ respectively. Since $\psi$ is continuous we may consider the direct image $\psi^{-1} \mathcal{F}_{2}$ of $\mathfrak{F}_{2}$ under $\psi$. If we define $\theta(U): \mathscr{F}_{1}(U) \rightarrow \psi^{-1} \mathfrak{F}_{2}(U)$ by $\theta(U)(f)$ $=f \psi$ we find the following holds.

LEMmA. $(\psi, \theta):\left(\mathcal{O}_{2}, F_{2}\right) \rightarrow\left(\mathcal{O}_{1}, \mathcal{F}_{1}\right)$ is a ringed space morphism.

Proof. We must show that for each $f \in \mathcal{F}_{1}(U),(\theta)(U)(f)$ is locally $\operatorname{good}$ on $\psi^{-1}(U)$. Then Proposition A finishes the proof. Let $\beta \in \psi^{-1}(U)$ then $\psi(\beta)=h^{-1}(\beta)=\alpha \in U$. Thus for $f \in \mathcal{F}_{1}(U)$ there exists $V$ open in $U$ and $b \in F_{1}$ such that $\alpha \in V$ and $f(\varphi)=(b, \varphi)$ for all $\varphi \in V$. Then $h(b)$ 
$\in F_{2}, \beta \in \psi^{-1}(V)$ and $\psi^{-1}(V)$ is open in $\psi^{-1}(U)$. Now for $\sigma \in \psi^{-1}(V)$ we have

$$
\begin{aligned}
(\theta(U)(f))(\sigma) & =f\left(h^{-1}(\sigma)\right)=\left(b, h^{-1}(\sigma)\right) \\
& =\sup \left\{r \in Q: b-r \in h^{-1}(\sigma)\right\} \\
& =\sup \left\{r \in Q: h(b)-h(r) \in h h^{-1}(\sigma)\right\} \\
& =\sup \left\{r \in Q: h(b)-r \in h h^{-1}(\sigma)\right\} \\
& =\sup \{r \in Q: h(b)-r \in \sigma\}=(h(b), \sigma) .
\end{aligned}
$$

The second to last equality holds because $Q \subseteq h h^{-1}(\sigma) \subseteq \sigma$. Thus $\theta(U)(f)$ is locally good on $\psi^{-1}(U)$.

Suppose $F_{1} \rightarrow^{h_{1}} F_{2} \rightarrow^{h_{2}} F_{3}, \psi_{1}: \Theta_{2} \rightarrow \Theta_{1}$ is induced by $h_{1}, \psi_{2}: \Theta_{3} \rightarrow \Theta_{2}$ is induced by $h_{2}$, and $\psi: \theta_{3} \rightarrow \Theta_{1}$ is induced by $h=h_{2} h_{1}$ then clearly $\psi=\psi_{1} \psi_{2}$. From [5] we know that ringed spaces and ringed space morphisms form a category with composition $\left(\psi_{1}, \theta_{1}\right)\left(\psi_{2}, \theta_{2}\right)=\left(\psi_{1} \circ \psi_{2}, \theta_{2} \circ \theta_{1}\right)$, $\psi_{1} \circ \psi_{2}$ the usual function composition and $\theta_{2} \circ \theta_{1}$ defined using the "direct image" functor. That is, $\theta_{2} \circ \theta_{1}=\left(\psi_{1}^{-1} \theta_{2}\right) \theta_{1}$.

LEMмA. If $\left(\psi_{1}, \theta_{1}\right),\left(\psi_{2}, \theta_{2}\right)$, and $(\psi, \theta)$ are the ringed space morphisms induced by $F_{1} \rightarrow^{h_{1}} F_{2}, \quad F_{2} \rightarrow^{h_{2}} F_{3}$, and $F_{1} \rightarrow^{h=h_{2} h_{1}} F_{3}$ respectively, then $(\psi, \theta)=\left(\psi_{1}, \theta_{1}\right)\left(\psi_{2}, \theta_{2}\right)$.

The proof is routine verification and hence omitted.

The previous three lemmas yield the following theorem.

Theorem 2. The map which associates with each field $F$ the ringed space $(\mathcal{O}, \mathcal{F})$ and with each field homomorphism $h$ the ringed space morphism $(\psi, \theta)$ is a contravariant functor from the category of fields and field homomorphisms to the category of ringed spaces and ringed space morphisms.

4. The sheaf $\bar{F}$. If $\alpha$ and $\beta$ are orders on a field $F$ we will say $\alpha$ is equivalent to $\beta$, denoted $\alpha \sim \beta$, if $(b, \alpha)=(b, \beta)$ for all $b \in F$. That is, if both $\alpha$ and $\beta$ determine the same place $F \rightarrow R$. $\mathcal{O} / \sim$ will denote the set of equivalence classes of orders on $F$ and $\varphi$ the canonical map $\theta \rightarrow \theta / \sim$. $\theta / \sim$ will be considered to have the induced quotient topology. If $\bar{\alpha} \in \mathcal{O} / \sim$ and $b \in F$ define $(b, \bar{\alpha})=(b, \alpha)$. It is easily seen that if $\alpha \sim \beta$ then $B_{\alpha}=B_{\beta}$ however the converse is not true. Thus for $\bar{\alpha} \in \mathcal{O} / \sim$ define $B_{\bar{\alpha}}=B_{\alpha}$.

We will now define locally good functions on open subsets of $\theta / \sim$ in a way similar to that for $\theta$. Let $\bar{U}$ be open in $\theta / \sim$. A real valued function $f$ on $\bar{U}$ is said to be locally good if for each $\bar{\alpha} \in \bar{U}$ there exists 
an open set $\bar{V} \subseteq \bar{U}$ and a $b \in F$ such that $\bar{\alpha} \in \bar{V}$ and $f(\bar{\beta})=(b, \bar{\beta})$ for all $\bar{\beta} \in \bar{V}$.

Proposition. If $f$ is locally good on $\bar{U}$ then $f \varphi$ is locally good on $\varphi^{-1} \bar{U}$.

Proof. If $\alpha \in \varphi^{-1}(\bar{U})$ then $\bar{\alpha} \in \bar{U}$ thus there exists $\bar{V}$ open in $\bar{U}, b \in F$, such that $\bar{\alpha} \in \bar{V}$ and $f(\bar{\beta})=(b, \bar{\beta})$ for all $\bar{\beta} \in \bar{V}$. Then $V=\varphi^{-1}(\bar{V})$ is open in $\varphi^{-1}(U), \alpha \in V$, and $f \varphi(\beta)=f(\bar{\beta})=(b, \bar{\beta})=(b, \beta)$ for all $\beta \in V$. Hence $f \varphi$ is locally good on $\varphi^{-1}(\bar{U})$.

For $\bar{U}$ open in $\mathcal{\theta} / \sim$ define $\bar{F}(\bar{U})=\{f: f$ is locally good on $\bar{U}\}$ and for $\bar{U} \subseteq \bar{V}$ open subsets of $\mathcal{O} / \sim$ define the restriction map $\bar{F}(\bar{V}) \rightarrow \bar{F}(\bar{U})$ to be the restriction map in the usual sense.

THEOREM 3. $\bar{F}$, defined above, is a sheaf of rings on $\mathcal{O} / \sim$.

The proof is omitted since it is exactly the same as the proof that $\mathfrak{F}$ is a sheaf of rings on $\theta$.

Let $F_{1} \rightarrow^{h} F_{2}$ be a field homomorphism and notice that $\alpha \sim \beta$ implies $h^{-1}(\alpha) \sim h^{-1}(\beta)$. Thus we can define $\bar{\psi}: \mathcal{O}_{2} / \sim \rightarrow \mathcal{O}_{1} / \sim$ by

$$
\bar{\psi}(\bar{\alpha})=\overline{h^{-1}(\alpha)} \text {. }
$$

Proposition. $\bar{\psi}$, defined above, is continuous.

Proof. Clearly $\varphi_{1} \psi_{1}=\psi \varphi_{2}$ where $\varphi_{1}, \varphi_{2}$ are the canonical maps $\Theta_{1} \rightarrow \theta_{1} / \sim, \theta_{2} \rightarrow \Theta_{2} / \sim$ respectively. $\varphi_{1}$ and $\psi$ are both continuous hence $\varphi_{1} \psi=\Psi \varphi_{2}$ is continuous. But $\mathcal{O}_{2} / \sim$ has the quotient topology induced by $\varphi_{2}$ and so $\Psi$ is continuous.

Continuing in the same manner as before we define $\bar{\theta}(\bar{U}): \bar{\Im}_{1}(\bar{U})$ $\rightarrow \psi^{-1} \overline{\mathcal{F}}_{2}(\bar{U})$ by $\bar{\theta}(\bar{U})(f)=f \bar{\psi}$ where $\bar{\psi}$ is considered to be restricted to $\psi^{-1}(\bar{U})$ and $\bar{F}_{1}$ and $\bar{F}_{2}$ are the sheaves on $\mathcal{O}_{1} / \sim$ and $\mathcal{O}_{2} / \sim$ respectively. Then, as in the previous section, $\bar{\theta}$ is a sheaf homomorphism $\bar{F}_{1}$ $\rightarrow \bar{\psi}^{-1} \overline{\mathfrak{F}}_{2}$ and we have the following theorem.

THEOREM 4. $(\bar{\psi}, \bar{\theta})$ is a morphism of ringed spaces and the map which associates with each field $F$ the sheaf $\overline{\mathcal{F}}$ and with each field homomorphism $h$ the ringed space morphism $(\Psi, \bar{\theta})$ is a contravariant functor from the category of fields to the category of ringed spaces.

The proof is omitted.

In view of the first proposition of this section and Proposition $\mathrm{A}, \S 2$ we can define $\pi(\bar{U}): \bar{F}(\bar{U}) \rightarrow \varphi^{-1} \bar{F}(\bar{U})$ by $\pi(U)(f)=f \varphi$ and we know that $(\varphi, \pi):(\mathcal{O}, \mathcal{F}) \rightarrow(\mathcal{O} / \sim, \overline{\mathcal{F}})$ is a ringed space morphism where $\pi$ $=\{\pi(\bar{U}): \bar{U}$ is open in $\mathcal{\theta} / \sim\}$. Denote by $(\varphi(F), \pi(F))$ the $(\varphi, \pi)$ just defined. 
Theorem 5. $\Pi=\{(\varphi(F), \pi(F)): F$ is a field $\}$ is a natural transformation from the functor of Theorem 2 to the functor of Theorem 4.

Proof. We must prove that if $F_{1} \rightarrow^{h} F_{2}$ is a field homomorphism then $\left(\varphi_{1}, \pi_{1}\right)(\psi, \theta)=(\psi, \bar{\theta})\left(\varphi_{2}, \pi_{2}\right)$ where $\left(\varphi_{1}, \pi_{1}\right)=\left(\varphi\left(F_{1}\right), \pi\left(F_{1}\right)\right)$ and $\left(\varphi_{2}, \pi_{2}\right)=\left(\varphi\left(F_{2}\right), \pi\left(F_{2}\right)\right)$. It is easily seen that $\varphi_{1} \psi=\bar{\psi} \varphi_{2}$ hence we need to prove that $\theta \pi_{1}=\pi_{2} \bar{\theta}$. That is $\left(\varphi_{1}^{-1} \theta\right) \pi_{1}=\left(\bar{\psi}^{-1} \pi_{2}\right) \bar{\theta}$. Thus for each $\bar{U}$ open in $\mathcal{O}_{1} / \sim$ we must prove that $\varphi_{1}^{-1} \theta(\bar{U}) \circ \pi_{1}(\bar{U})=\psi_{1}^{-1} \pi_{2}(\bar{U})$ $\circ \bar{\theta}(\bar{U})$ but this is routine.

EXAMPLE. Let $Q$ be the field of rational numbers and $x$ an indeterminant. From [2] we know that $Q(x)$ is a field with the property that $\alpha \sim \beta$ if and only if $(x, \alpha)=(x, \beta)$ and that $f_{x}$ defined by $f_{x}(\bar{\beta})=(x, \bar{\beta})$ is a continuous one-to-one function from $\mathcal{O} / \sim$ onto $R^{\prime}$ where $R^{\prime}$ is the one point compactification of real numbers. Also, from [6] we know that $\mathcal{O}$ is compact and hence $\mathcal{O} / \sim$ is compact. Thus $f_{x}$ is a continuous one-to-one function from a compact space onto a Hausdorff space implying that $f_{x}$ is a homeomorphism. If $f \in Q(x)$ then $(f, \alpha)=$ $\sup \{r \in Q: f-r \in \alpha\}=\sup \{r \in Q: f-r>(x, \alpha)\}=f((x, \alpha))=f\left(f_{x}(\alpha)\right)$. Thus we know that if we identify $\Theta / \sim$ with $R^{\prime}$ and let $U$ be open then a function is locally good on $U$ if and only if it is a finite valued rational function on each connected component of $U$.

The ring homomorphism $\pi(U)$ is clearly injective; however, it is not surjective. Let $\alpha \neq \beta$ be such that $\alpha \sim \beta$, then there exists $b \in F$ such that $b \in \alpha$ and $-b \in \beta$. Define $U_{\alpha}=\{\sigma \in \mathcal{O}:-b \in \sigma\}$ and $U_{\beta}$ $=\{\sigma \in \mathcal{\theta}: b \in \sigma\} . U_{\alpha}$ and $U_{\beta}$ form a disconnection of the space $\theta$ with $\alpha \in U_{\alpha}$ and $\beta \in U_{\beta}$. Now define $f: \Theta \rightarrow R$ by $f(\sigma)=(1, \sigma)=1$ if $\sigma \in U_{\alpha}$ and $f(\sigma)=(2, \sigma)=2$ if $\sigma \in U_{\beta}$. Then $f \in \mathcal{F}(\mathcal{\theta})$ and $f(\alpha) \neq f(\beta)$ thus there does not exist $g \in \bar{F}(\mathcal{O} / \sim)$ such that $g \varphi=f$. From this example we see that $\pi$ is a natural isomorphism if and only if $\alpha \sim \beta$ implies $\alpha=\beta$.

5. Spec $B$. Let $A$ be a commutative ring. Spec $A=Y$ is the set of all prime ideals in $A$. For $f \in A$ define $D(f)=\{p \in \operatorname{Spec} A: f \notin p\}$ then the set of $D(f)$ is a basis for the Zariski topology on Spec $A$. If $f, g$ in $A$ are such that $D(f) \supseteq D(g)$ then there exists $n>0$ and $s \in A$ such that $g^{n}=s f$. Define $\rho_{g, f}: A_{f} \rightarrow A_{g}$ by $\rho_{g, f}\left(a / f^{m}\right)=a s / g^{m n}$ where $A_{f}$ is the ring of fractions of $A$ with respect to $\left\{f^{n}: n \geqq 0\right\}$. This association $D(f) \rightarrow A_{f}$ defines a presheaf on the basis $\{D(f): f \in A\}$ for the Zariski topology and hence determines a presheaf on $Y$ which is actually a sheaf $\theta_{Y}$ on $Y$ called the structure sheaf [8].

If $\alpha$ is an order on a field $F$ let $B_{\alpha}$ denote the valuation ring corresponding to $\alpha$ called the ring of bounded elements, $J$ denote the ring of infinitesimals and $B=\bigcap\left\{\beta_{\alpha}: \alpha \in \mathcal{O}\right\}$. For $X=\mathcal{O} / \sim$ and $Y=\operatorname{Spec} B$ define $\psi: X \rightarrow Y \quad$ by $\quad \psi(\bar{\alpha})=J_{\alpha} \cap B . \quad \psi^{-1}(D(f))=\left\{\alpha \in X: f \notin J_{\alpha}\right\}$ 
$=\{\alpha \in X:(f, \alpha) \neq 0\}$ which is open since $(f, ?)$ is continuous thus $\psi$ is continuous. If we now consider the sheaf $\psi^{-1} \overline{\mathfrak{F}}$ we can define $\theta_{f}$ : $\hat{O}_{Y}(D(f))=B_{f} \rightarrow \psi^{-1} \overline{\mathcal{F}}(D(f))$ by $\theta_{f}\left(b / f^{m}\right)=(b, ?) /(f, ?)^{m}$ restricted to $\psi^{-1}(D(f))$. It is easy to verify that $\theta_{f}\left(b / f^{m}\right)$ is locally good on $\psi^{-1}(D(f))$ and that $\theta_{f}$ is a ring homomorphism.

THEOREM 6. $\theta$ determined by $\left\{\theta_{f}: f \in \beta\right\}$ is a sheaf homomorphism $\hat{O}_{Y} \rightarrow \psi^{-1} \overline{\mathcal{F}}$ and hence $(\psi, \theta)$ is a morphism of ringed spaces.

\section{REFERENCES}

1. Glen E. Bredon, Sheaf theory, McGraw-Hill, New York, 1967. MR 36 \#552.

2. D. W. Dubois, Infinite primes and ordered fields, Dissertationes Math. 69 (1970).

3. Samuel Eilenberg and Norman Steenrod, Foundations of algebraic topology, Princeton Univ. Press, Princeton, N. J., 1952. MR 14, 398.

4. Roger Godement, Théorie des faisceaux, Publ. Inst. Math. Univ. Strasbourg, Hermann, Paris, 1958.

5. A. Grothendieck, Éléments de géométrie algébrique, Inst. Hautes Etudes Sci. Publ. Math. No. 4 (1960).

6. D. K. Harrison, Finite and infinite primes for rings and fields, Mem. Amer. Math. Soc. No. 68 (1966). MR 34 \#7550.

7. John L. Kelly, General topology, Van Nostrand, Princeton, N. J., 1955. MR 16, 1136.

8. I. G. MacDonald, Algebraic geometry. Introduction to schemes, Benjamin, New York, 1968. MR 39 \#205.

University of New Mexico, Albuquerque, New Mexico 87105

University of Pittsburgh, Pittsburgh, Pennsylvania 15213 